\title{
Semiflow selection for the compressible Navier-Stokes system
}

\author{
DANICA BASARIĆ
}

Abstract. Although the existence of dissipative weak solutions for the compressible Navier-Stokes system has already been established for any finite energy initial data, uniqueness is still an open problem. The idea is then to select a solution satisfying the semigroup property, an important feature of systems with uniqueness. More precisely, we are going to prove the existence of a semiflow selection in terms of the three state variables: the density, the momentum, and the energy. Finally, we will show that it is possible to introduce a new selection defined only in terms of the initial density and momentum; however, the price to pay is that the semigroup property will hold almost everywhere in time.

\section{Introduction}

Consider the compressible Navier-Stokes system

$$
\begin{aligned}
& \partial_{t} \varrho+\operatorname{div}_{x}(\varrho \mathbf{u})=0, \\
& \partial_{t}(\varrho \mathbf{u})+\operatorname{div}_{x}(\varrho \mathbf{u} \otimes \mathbf{u})+\nabla_{x} p(\varrho)=\operatorname{div}_{x} \mathbb{S}\left(\nabla_{x} \mathbf{u}\right),
\end{aligned}
$$

where $\varrho=\varrho(t, x)$ denotes the density, $\mathbf{u}=\mathbf{u}(t, x)$ the velocity, $p=p(\varrho)$ the pressure, and $\mathbb{S}=\mathbb{S}\left(\nabla_{x} \mathbf{u}\right)$ the viscous stress. We will consider the system on the set $(t, x) \in(0, \infty) \times \Omega$, where $\Omega \subset \mathbb{R}^{N}, N=2,3$ is a bounded domain with $\partial \Omega$ of class $C^{2+v}$ for a certain $v>0$. As our goal is to handle a potentially ill-posed problem, we have deliberately omitted the case $N=1$, for which the problem is known to be well posed, see Kazhikhov [8].

We impose the no-slip boundary condition for the velocity

$$
\left.\mathbf{u}\right|_{\partial \Omega}=0 \text { for all } t \in[0, \infty),
$$

and we prescribe the initial conditions

$$
\varrho(0, \cdot)=\varrho_{0}, \quad(\varrho \mathbf{u})(0, \cdot)=(\varrho \mathbf{u})_{0} .
$$

Finally, we assume a barotropic pressure $p \in C[0, \infty) \cap C^{1}(0, \infty)$ such that $p(0)=0$ and

$$
\begin{cases}p^{\prime}(\varrho) \geq a_{1} \varrho^{\gamma-1}-b & \text { for all } \varrho>0 \\ p(\varrho) \leq a_{2} \varrho^{\gamma}+b & \text { for all } \varrho \geq 0\end{cases}
$$


for certain constants $a_{1}>0, a_{2}$ and $b$, with $\gamma>\frac{N}{2}$ the adiabatic exponent, and the viscous stress tensor to be a linear function of the velocity gradient, more specifically to satisfy the Newton's rheological law

$$
\mathbb{S}\left(\nabla_{x} \mathbf{u}\right)=\mu\left(\nabla_{x} \mathbf{u}+\nabla_{x}^{T} \mathbf{u}-\frac{2}{N}\left(\operatorname{div}_{x} \mathbf{u}\right) \mathbb{I}\right)+\lambda\left(\operatorname{div}_{x} \mathbf{u}\right) \mathbb{I},
$$

with $\mu>0$ and $\lambda \geq 0$. We would like to point out that (5) allows the pressure to be a general non-monotone function of the density, besides the standard case $p(\varrho)=a \varrho^{\gamma}$ with $a>0$ and $\gamma>\frac{N}{2}$. Still, as we shall see below, the problem admits global-intime weak solutions and retains other fundamental properties of the system, notably the weak-strong uniqueness, see [5].

We will consider dissipative weak solutions, i.e., solutions satisfying Equations (1) and (2) in a distributional sense along with the energy inequality, see Sect. 1.1. Although the existence of global in time solutions has already been established for any finite energy initial data, see, e.g., [10] and [6], uniqueness is still an open task. Then, a natural question is whether it is possible or not to select a solution satisfying at least the semiflow property, an important feature of systems with uniqueness: letting the system run from time 0 to time $s$ and then restarting and letting it run from time $s$ to time $t$ gives the same outcome as letting it run directly from time 0 to time $t$.

The result presented in this manuscript can be seen as the deterministic version of the stochastic paper done by Breit, Feireisl and Hofmanová [2]. The construction of the semigroup arises from the theory of Markov selection in order to study the well-posedness of certain systems; it was first developed by Krylov [9] and later adapted by Flandoli and Romito [7], Cardona and Kapitanski [4] in the context of the incompressible Navier-Stokes system.

Breit, Feireisl, and Hofmanová [3] used the deterministic version motivated by [4] to show the existence of the semiflow selection for dissipative measure-valued solutions of the isentropic Euler system. Following the same strategy, we will establish the existence of a semiflow selection for the compressible Navier-Stokes system (1)(6). Specifically, introducing the momentum $\mathbf{m}=\varrho \mathbf{u}$, we show the existence of a measurable mapping

$$
V:\left[t, \varrho_{0}, \mathbf{m}_{0}\right] \mapsto[\varrho(t), \mathbf{m}(t)], \quad t \geq 0,
$$

satisfying the semigroup property:

$$
V\left[t_{1}+t_{2}, \varrho_{0}, \mathbf{m}_{0}\right]=V\left[t_{2}, V\left[t_{1}, \varrho_{0}, \mathbf{m}_{0}\right]\right] \text { for a.e. } t_{1}, t_{2} \geq 0,
$$

where $[\varrho, \mathbf{m}=\varrho \mathbf{u}]$ represents a dissipative weak solution to (1)-(6). At this stage, we would like to point out the main essential difference between the present paper and [3]. The semigroup constructed for the Euler system in [3] contains the total energy as one of the state variables. This may be seen as a kind of drawback as the energy should be determined in terms of the basic state variables $[\varrho, \mathbf{m}]$. This is, however, a delicate issue for the Euler flow as the energy contains also the defect due to possible concentrations 
and/or oscillations. Such a problem does not occur for the Navier-Stokes system, where the energy is indeed a function of $[\varrho, \mathbf{u}]$ at least for a.a. $t \in[0, \infty)$, cf. (7).

The paper is organized as follows: The remaining part of this section contains the definitions of a dissipative weak solution and admissibility. In Sect. 2, we fix the topologies on the space of the initial data and the trajectory space, and we introduce the concept of a semiflow selection in terms of the three state variables: the density $\varrho_{0}$, the momentum $\mathbf{m}_{0}$, and the energy $E_{0}$. In Sect. 3, we analyze the properties (compactness, non-emptiness, the shift invariance, and continuation properties) of the solution set for a given initial data, while Sect. 4 is devoted to the proof of the existence of a semiflow selection. Finally, in Sect. 5, we study a new selection defined only in terms of the initial density $\varrho_{0}$ and the momentum $\mathbf{m}_{0}$.

1.1. Dissipative weak solution

Following [6], we can give the definition of a dissipative solution to the compressible Navier-Stokes system.

Definition 1.1. The pair of functions $\varrho, \mathbf{u}$ is called dissipative weak solution of the Navier-Stokes system (1)-(6) with the total energy $E$ and initial data

$$
\left[\varrho_{0},(\varrho \mathbf{u})_{0}, E_{0}\right] \in L^{\gamma}(\Omega) \times L^{\frac{2 \gamma}{\gamma+1}}\left(\Omega ; \mathbb{R}^{N}\right) \times[0, \infty)
$$

if the following holds:

(i) regularity class:

$[\varrho, \varrho \mathbf{u}, E] \in C_{\text {weak }, \text { loc }}\left([0, \infty) ; L^{\gamma}(\Omega)\right) \times C_{\text {weak }, \text { loc }}\left([0, \infty) ; L^{\frac{2 \gamma}{\gamma+1}}\left(\Omega ; \mathbb{R}^{N}\right)\right)$

$$
\times B V_{\mathrm{loc}}([0, \infty)) \text {, }
$$

with $\varrho \geq 0$;

(ii) weak formulation of the renormalized continuity equation: for any $\tau>0$ and any functions

$$
\begin{aligned}
& B \in C[0, \infty) \cap C^{1}(0, \infty), b \in C[0, \infty) \text { bounded on }[0, \infty), \\
& B(0)=b(0)=0 \text { and } b(z)=z B^{\prime}(z)-B(z) \text { for any } z>0,
\end{aligned}
$$

the integral identity

$$
\begin{aligned}
{\left[\int_{\Omega} B(\varrho) \varphi(t, \cdot) \mathrm{d} x\right]_{t=0}^{t=\tau}=} & \int_{0}^{\tau} \int_{\Omega}\left[B(\varrho) \partial_{t} \varphi\right. \\
& \left.+B(\varrho) \mathbf{u} \cdot \nabla_{x} \varphi+b(\varrho) \operatorname{div}_{x} \mathbf{u} \varphi\right] d x d t
\end{aligned}
$$

holds for any $\varphi \in C_{c}^{1}([0, \infty) \times \Omega)$;

(iii) weak formulation of the balance of momentum: for any $\tau>0$ the integral identity

$$
\left[\int_{\Omega} \varrho \mathbf{u} \cdot \boldsymbol{\varphi}(t, \cdot) \mathrm{d} x\right]_{t=0}^{t=\tau}=\int_{0}^{\tau} \int_{\Omega}\left[\varrho \mathbf{u} \cdot \partial_{t} \boldsymbol{\varphi}+(\varrho \mathbf{u} \otimes \mathbf{u}): \nabla_{x} \boldsymbol{\varphi}\right.
$$




$$
\left.+p(\varrho) \operatorname{div}_{x} \boldsymbol{\varphi}-\mathbb{S}\left(\nabla_{x} \mathbf{u}\right): \nabla_{x} \boldsymbol{\varphi}\right] d x d t
$$

holds for any $\boldsymbol{\varphi} \in C_{c}^{1}\left([0, \infty) \times \Omega ; \mathbb{R}^{N}\right)$, where $(\varrho \mathbf{u})(0, \cdot)=(\varrho \mathbf{u})_{0} ;$

(iv) energy inequality: for a.e. $\tau \geq 0$ we have

$$
E(\tau)=\int_{\Omega}\left[\frac{1}{2} \varrho|\mathbf{u}|^{2}+P(\varrho)\right](\tau, \cdot) \mathrm{d} x
$$

where the pressure potential $P$ is chosen as a solution of

$$
\varrho P^{\prime}(\varrho)-P(\varrho)=p(\varrho)
$$

we also require $E=E(\tau)$ to be a non-increasing function of $\tau$ :

$$
[E \psi]_{t=\tau_{1}-}^{t=\tau_{2}+}-\int_{\tau_{1}}^{\tau_{2}} E(t) \psi^{\prime}(t) \mathrm{d} t+\int_{\tau_{1}}^{\tau_{2}} \psi \int_{\Omega} \mathbb{S}\left(\nabla_{x} \mathbf{u}\right): \nabla_{x} \mathbf{u} d x d t \leq 0,
$$

for any $0 \leq \tau_{1} \leq \tau_{2}, \psi \in C_{c}^{1}[0, \infty), \psi \geq 0$, where $E(0-)=E_{0}$.

Remark 1.2. Condition (ii) can be considered as a simple rescaling of the state variables in the continuity equation (1); it is necessary in order to prove the weak sequential stability and the existence of dissipative weak solutions. In particular, choosing $B(z)=z$ we get the standard weak formulation of the continuity equation.

Remark 1.3. At this stage, similarly to [3], the total energy is considered as an additional phase variable - a non-increasing function of time possessing one sided limits at any time. In contrast with [3], the energy can be determined in terms of $\varrho$ and $\mathbf{u}$, see (10), with the exception of a zero measure set of times.

Remark 1.4. The condition $E(0-)=E_{0}$ comes naturally from the assumption that the total energy is bounded at the initial time $t=0$, specifically

$$
E(0+) \leq E_{0}
$$

\subsection{Admissible solution}

From now on, it is more convenient to work with the momentum $\mathbf{m}=\varrho \mathbf{u}$. Following [3], we introduce a subclass of dissipative solutions that reflect the physical principle of minimization of the total energy. At the present state, we retain the total energy $E$ as an integral part of the solution so we work with the triples $[\varrho, \mathbf{m}, E]$. Finally, in Sect. 5, we pass to the natural state variables $[\varrho, \mathbf{m}]$. To this end, let $\left[\varrho^{i}, \mathbf{m}^{i}, E^{i}\right]$, $i=1,2$, be two dissipative solutions starting from the same initial data $\left[\varrho_{0}, \mathbf{m}_{0}, E_{0}\right]$. We introduce the relation

$$
\left[\varrho^{1}, \mathbf{m}^{1}, E^{1}\right] \prec\left[\varrho^{2}, \mathbf{m}^{2}, E^{2}\right] \Leftrightarrow E^{1}(\tau \pm) \leq E^{2}(\tau \pm) \text { for any } \tau \in(0, \infty) \text {. }
$$


Definition 1.5. We say that a dissipative weak solution $[\varrho, \mathbf{m}, E]$ starting from the initial data $\left[\varrho_{0}, \mathbf{m}_{0}, E_{0}\right]$ is admissible if it is minimal with respect to the relation $\prec$. Specifically, if

$$
[\tilde{\varrho}, \tilde{\mathbf{m}}, \tilde{E}] \prec[\varrho, \mathbf{m}, E],
$$

where $[\tilde{\varrho}, \tilde{\mathbf{m}}, \tilde{E}]$ is another dissipative solution starting from $\left[\varrho_{0}, \mathbf{m}_{0}, E_{0}\right]$, then

$$
E=\tilde{E} \text { in }[0, \infty)
$$

In particular, such selection criterion guarantees that equilibrium states belong to the class of dissipative weak solutions (see [3], Section 6.3).

\section{Setup}

We start by introducing suitable topologies on the space of initial data and the space of dissipative weak solutions. Fix $\ell>\frac{N}{2}+1$ and for simplicity consider the Hilbert space

$$
X=W^{-\ell, 2}(\Omega) \times W^{-\ell, 2}\left(\Omega ; \mathbb{R}^{N}\right) \times \mathbb{R}
$$

together with its subset containing the initial data

$$
\begin{aligned}
D= & \left\{\left[\varrho_{0}, \mathbf{m}_{0}, E_{0}\right] \in X: \varrho_{0} \in L^{1}(\Omega), \varrho_{0} \geq 0, \mathbf{m}_{0} \in L^{1}\left(\Omega ; \mathbb{R}^{N}\right),\right. \\
& \left.\int_{\Omega}\left[\frac{1}{2} \frac{\left|\mathbf{m}_{0}\right|^{2}}{\varrho_{0}}+P\left(\varrho_{0}\right)\right] \mathrm{d} x \leq E_{0}\right\} .
\end{aligned}
$$

Here, the convex function $[\varrho, \mathbf{m}] \mapsto \frac{|\mathbf{m}|^{2}}{\varrho}$ is defined for $\varrho \geq 0, \mathbf{m} \in \mathbb{R}^{N}$ as

$$
\frac{|\mathbf{m}|^{2}}{\varrho}= \begin{cases}0 & \text { if } \mathbf{m}=0, \\ \frac{|\mathbf{m}|^{2}}{\varrho} & \text { if } \varrho>0, \\ \infty & \text { otherwise }\end{cases}
$$

If $\left[\varrho_{0}, \mathbf{m}_{0}, E_{0}\right] \in D$, applying Hölder inequality, we can also deduce that $\varrho_{0} \in L^{\gamma}(\Omega)$ and $\mathbf{m}_{0} \in L^{\frac{2 \gamma}{\gamma+1}}\left(\Omega ; \mathbb{R}^{N}\right)$; accordingly, the set of the data can be seen as a closed convex subset of the Banach space $L^{\gamma}(\Omega) \times L^{\frac{2 \gamma}{\gamma+1}}\left(\Omega ; \mathbb{R}^{N}\right) \times \mathbb{R}$. Indeed, denoting with $L_{+}^{1}(\Omega)$ the space of non-negative integrable functions, we can write

$$
D=\left\{\left[\varrho_{0}, \mathbf{m}_{0}, E_{0}\right] \in L_{+}^{1}(\Omega) \times L^{1}\left(\Omega ; \mathbb{R}^{N}\right) \times \mathbb{R}: f\left(\left[\varrho_{0}, \mathbf{m}_{0}\right]\right) \leq E_{0}\right\},
$$

so that it coincides with the epigraph of the function $f: L_{+}^{1}(\Omega) \times L^{1}\left(\Omega ; \mathbb{R}^{N}\right) \rightarrow$ $[0,+\infty]$ such that

$$
f\left(\left[\varrho_{0}, \mathbf{m}_{0}\right]\right)=\int_{\Omega}\left[\frac{1}{2} \frac{\left|\mathbf{m}_{0}\right|^{2}}{\varrho_{0}}+P\left(\varrho_{0}\right)\right] \mathrm{d} x .
$$


Since $f$ is lower semi-continuous and convex, we obtain that its epigraph is closed and convex.

We consider the trajectory space

$$
Q=C_{\mathrm{loc}}\left([0, \infty) ; W^{-\ell, 2}(\Omega)\right) \times C_{\mathrm{loc}}\left([0, \infty) ; W^{-\ell, 2}\left(\Omega ; \mathbb{R}^{N}\right)\right) \times L_{\mathrm{loc}}^{1}[0, \infty),
$$

which is a separable space. Dissipative weak solutions $[\varrho, \mathbf{m}, E]$, as defined in Definition 1.1 , belong to this class - since $\ell>\frac{N}{2}$, we have that $L^{p}$ with $p \geq 1$ is compactly embedded in $W^{-\ell, 2}$ so in particular it holds for $p=\gamma$ and $p=\frac{2 \gamma}{\gamma+1}$-while Equations (8) and (9) give an information on the time regularity of the density and the momentum. Moreover, for any initial data $\left[\varrho_{0}, \mathbf{m}_{0}, E_{0}\right] \in D$, it follows that a dissipative weak solution $[\varrho, \mathbf{m}, E]$ evaluated at every $t \geq 0$ also belongs to the set $D$, due to the energy inequality. Finally, for initial data $\left[\varrho_{0}, \mathbf{m}_{0}, E_{0}\right] \in D$, we introduce the solution set

$\mathcal{U}\left[\varrho_{0}, \mathbf{m}_{0}, E_{0}\right]$

$=\{[\varrho, \mathbf{m}, E] \in Q \mid[\varrho, \mathbf{m}=\varrho \mathbf{u}, E]$ is a dissipative weak solution with initial data $\left.\left[\varrho_{0}, \mathbf{m}_{0}, E_{0}\right]\right\}$.

\subsection{Semiflow selection: main result}

We are now ready to define a semiflow selection to (1)-(6).

Definition 2.1. A semiflow selection in the class of dissipative weak solutions for the compressible Navier-Stokes system (1)-(6) is a mapping

$$
U: D \rightarrow Q, \quad U\left\{\varrho_{0}, \mathbf{m}_{0}, E_{0}\right\} \in \mathcal{U}\left[\varrho_{0}, \mathbf{m}_{0}, E_{0}\right] \text { for any }\left[\varrho_{0}, \mathbf{m}_{0}, E_{0}\right] \in D
$$

enjoying the following properties:

(i) Measurability. The mapping $U: D \rightarrow Q$ is Borel measurable.

(ii) Semigroup property. We have

$$
U\left\{\varrho_{0}, \mathbf{m}_{0}, E_{0}\right\}\left(t_{1}+t_{2}\right)=U\left\{\varrho\left(t_{1}\right), \mathbf{m}\left(t_{1}\right), E\left(t_{1}-\right)\right\}\left(t_{2}\right),
$$

where $[\varrho, \mathbf{m}, E]=U\left\{\varrho_{0}, \mathbf{m}_{0}, E_{0}\right\}$ for any $\left[\varrho_{0}, \mathbf{m}_{0}, E_{0}\right] \in D$ and any $t_{1}, t_{2} \geq 0$.

We are now ready to state our main result; the proof is postponed to Sect. 4.1.

Theorem 2.2. The compressible Navier-Stokes system (1)-(6) admits a semiflow selection $U$ in the class of dissipative weak solutions in the sense of Definition 2.1. Moreover, we have that $U\left\{\varrho_{0}, \boldsymbol{m}_{0}, E_{0}\right\}$ is admissible in the sense of Definition 1.5, for any $\left[\varrho_{0}, \boldsymbol{m}_{0}, E_{0}\right] \in D$.

Theorem 2.2 is stated in terms of the three state variables $[\varrho, \mathbf{m}, E]$. In Sect. 5, we state a version of this result in terms of the natural state variables $[\varrho, \mathbf{m}]$, see Theorem 5.1. The price to pay is validity of the semigroup property for any time with the exception of a zero measure set. 


\section{Properties of $\mathcal{U}$}

3.1. Existence and sequential stability

First of all, we aim to show:

1. existence of a dissipative weak solution for any initial data $\left[\varrho_{0}, \mathbf{m}_{0}, E_{0}\right] \in D$, meaning

$$
\mathcal{U}\left[\varrho_{0}, \mathbf{m}_{0}, E_{0}\right] \neq \emptyset
$$

2. weak sequential stability of the solution set, meaning

$$
\mathcal{U}\left[\varrho_{0}, \mathbf{m}_{0}, E_{0}\right] \subset Q \text { is compact }
$$

and the multivalued mapping

$$
\left[\varrho_{0}, \mathbf{m}_{0}, E_{0}\right] \in D \subset X \rightarrow \mathcal{U}\left[\varrho_{0}, \mathbf{m}_{0}, E_{0}\right] \in 2^{Q}
$$

has closed graph; in particular, this implies that it is continuous with respect to the Hausdorff complementary topology and thus Borel measurable, see [3] for details.

Both results can be found in [6], see Theorems 6.1, 6.2 and 7.1 for details.

Proposition 3.1. Suppose that $\left\{\varrho_{0, \varepsilon}, \boldsymbol{m}_{0, \varepsilon}, E_{0, \varepsilon}\right\}_{\varepsilon>0} \subset D$ is a sequence of data giving rise to a family of dissipative weak solutions $\left\{\varrho_{\varepsilon}, \boldsymbol{m}_{\varepsilon}, E_{\varepsilon}\right\}_{\varepsilon>0}$, that is, $\left[\varrho_{\varepsilon}, \boldsymbol{m}_{\varepsilon}, E_{\varepsilon}\right] \in$ $\mathcal{U}\left[\varrho_{0, \varepsilon}, \boldsymbol{m}_{0, \varepsilon}, E_{0, \varepsilon}\right]$. Moreover, we assume that the initial densities converge strongly

$$
\varrho_{0, \varepsilon} \rightarrow \varrho_{0} \text { in } L^{\gamma}(\Omega)
$$

and there exists a constant $\bar{E}>0$ such that $E_{0, \varepsilon} \leq \bar{E}$ for all $\varepsilon>0$.

Then, at least for suitable subsequences,

$$
\boldsymbol{m}_{0, \varepsilon} \rightarrow \boldsymbol{m}_{0} \quad \text { in } L^{\frac{2 \gamma}{\gamma+1}}\left(\Omega ; \mathbb{R}^{N}\right), \quad E_{0, \varepsilon} \rightarrow E_{0},
$$

and

$$
\begin{gathered}
\varrho_{\varepsilon} \rightarrow \varrho \text { in } C_{\text {weak, loc }}\left([0, \infty) ; L^{\gamma}(\Omega)\right) \\
\boldsymbol{m}_{\varepsilon} \rightarrow \boldsymbol{m} \text { in } C_{\text {weak }, \operatorname{loc}}\left([0, \infty) ; L^{\frac{2 \gamma}{\gamma+1}}\left(\Omega ; \mathbb{R}^{N}\right)\right) \\
E_{\varepsilon}(\tau) \rightarrow E(\tau) \text { for every } \tau \in[0, \infty) \text { and in } L_{\text {loc }}^{1}(0, \infty),
\end{gathered}
$$

where

$$
[\varrho, \boldsymbol{m}, E] \in \mathcal{U}\left[\varrho_{0}, \boldsymbol{m}_{0}, E_{0}\right]
$$

Proposition 3.2. Let $\left[\varrho_{0}, \boldsymbol{m}_{0}, E_{0}\right] \in D$ be given; then, the Navier-Stokes system (1)(6) admits a dissipative weak solution in the sense of Definition 1.1 with the initial data $\left[\varrho_{0}, \boldsymbol{m}_{0}, E_{0}\right]$. 
The following statement is a direct consequence of Propositions 3.1 and 3.2.

Lemma 3.3. For any $\left[\varrho_{0}, \boldsymbol{m}_{0}, E_{0}\right] \in D$, the set $\mathcal{U}\left[\varrho_{0}, \boldsymbol{m}_{0}, E_{0}\right]$ is a non-empty, compact subset of $Q$. Moreover, $[\varrho(T), \boldsymbol{m}(T), \mathcal{E}] \in D$ for any $T>0$, and for arbitrary $\mathcal{E} \geq E(T+)$.

\subsection{Shift and continuation operations}

In order to construct the semiflow, two main ingredients are needed: the shift invariance property and the continuation. For $\omega \in Q$, we define the positive shift operator

$$
S_{T} \circ \omega, S_{T} \circ \omega(t)=\omega(T+t), t \geq 0 .
$$

Lemma 3.4. Let $\left[\varrho_{0}, \boldsymbol{m}_{0}, E_{0}\right] \in D$ and $[\varrho, \boldsymbol{m}, E] \in \mathcal{U}\left[\varrho_{0}, \boldsymbol{m}_{0}, E_{0}\right]$. Then, we have

$$
S_{T} \circ[\varrho, \boldsymbol{m}, E] \in \mathcal{U}[\varrho(T), \boldsymbol{m}(T), \mathcal{E}]
$$

for any $T>0$, and any $\mathcal{E} \geq E(T+)$.

Proof. Obviously, a dissipative weak solution on the time interval $(0, \infty)$ solves also the same problem on $(T, \infty)$ with the initial data $[\varrho(T), \mathbf{m}(T), E(T+)]$. Shifting the test functions in the integrals, this implies

$$
S_{T} \circ[\varrho, \mathbf{m}, E] \in \mathcal{U}[\varrho(T), \mathbf{m}(T), E(T+)] .
$$

Since the energy is non-increasing, we can choose every $\mathcal{E} \geq E(T+)$ as initial energy; indeed, everything will be well defined

$$
S_{T} \circ E(0-)=\mathcal{E} \geq E(T+) .
$$

Remark 3.5. Since the energy is non-increasing, one can choose $\mathcal{E}=E(T-) \geq$ $E(T+)$.

For $\omega_{1}, \omega_{2} \in Q$ we define the continuation operator $\omega_{1} \cup_{T} \omega_{2}$ by

$$
\omega_{1} \cup_{T} \omega_{2}(t)= \begin{cases}\omega_{1}(t) & \text { for } 0 \leq t \leq T, \\ \omega_{2}(t-T) & \text { for } t>T .\end{cases}
$$

Lemma 3.6. Let $\left[\varrho_{0}, \boldsymbol{m}_{0}, E_{0}\right] \in D$ and

$$
\left[\varrho^{1}, \boldsymbol{m}^{1}, E^{1}\right] \in \mathcal{U}\left[\varrho_{0}, \boldsymbol{m}_{0}, E_{0}\right],\left[\varrho^{2}, \boldsymbol{m}^{2}, E^{2}\right] \in \mathcal{U}\left[\varrho^{1}(T), \boldsymbol{m}^{1}(T), \mathcal{E}\right],
$$

for some $\mathcal{E} \leq E^{1}(T-)$. Then,

$$
\left[\varrho^{1}, \boldsymbol{m}^{1}, E^{1}\right] \cup_{T}\left[\varrho^{2}, \boldsymbol{m}^{2}, E^{2}\right] \in \mathcal{U}\left[\varrho_{0}, \boldsymbol{m}_{0}, E_{0}\right] .
$$

Proof. We are simply pasting two solutions together at the time $T$, letting the second start from the point reached by the first one at the time $T$; thus, the integral identities remain satisfied. Choosing the initial energy for $\left[\varrho^{2}, \mathbf{m}^{2}, E^{2}\right]$ less or equal $E^{1}(T-)$, the energy of the solution $\left[\varrho^{1}, \mathbf{m}^{1}, E^{1}\right] \cup_{T}\left[\varrho^{2}, \mathbf{m}^{2}, E^{2}\right]$ remains non-increasing on $(0, \infty)$. 


\subsection{General ansatz}

Summarizing the results achieved in this section, we have shown the existence of a set-valued mapping

$$
D \ni\left[\varrho_{0}, \mathbf{m}_{0}, E_{0}\right] \mapsto \mathcal{U}\left[\varrho_{0}, \mathbf{m}_{0}, E_{0}\right] \in 2^{Q}
$$

enjoying the following properties.

(A1) Compactness: for any $\left[\varrho_{0}, \mathbf{m}_{0}, E_{0}\right] \in D$, the set $\mathcal{U}\left[\varrho_{0}, \mathbf{m}_{0}, E_{0}\right]$ is a non-empty compact subset of $Q$.

(A2) Measurability: the mapping

$$
D \ni\left[\varrho_{0}, \mathbf{m}_{0}, E_{0}\right] \mapsto \mathcal{U}\left[\varrho_{0}, \mathbf{m}_{0}, E_{0}\right] \in 2^{Q}
$$

is Borel measurable, where the range of $\mathcal{U}$ is endowed with the Hausdorff metric on the subspace of compact sets in $2^{Q}$.

(A3) Shift invariance: for any $[\varrho, \mathbf{m}, E] \in \mathcal{U}\left[\varrho_{0}, \mathbf{m}_{0}, E_{0}\right]$, we have

$$
S_{T} \circ[\varrho, \mathbf{m}, E] \in \mathcal{U}[\varrho(T), \mathbf{m}(T), E(T-)],
$$

for any $T>0$.

(A4) Continuation: if $T>0$, and

$$
\left[\varrho^{1}, \mathbf{m}^{1}, E^{1}\right] \in \mathcal{U}\left[\varrho_{0}, \mathbf{m}_{0}, E_{0}\right],\left[\varrho^{2}, \mathbf{m}^{2}, E^{2}\right] \in \mathcal{U}\left[\varrho^{1}(T), \mathbf{m}^{1}(T), E^{1}(T-)\right],
$$

then

$$
\left[\varrho^{1}, \mathbf{m}^{1}, E^{1}\right] \cup_{T}\left[\varrho^{2}, \mathbf{m}^{2}, E^{2}\right] \in \mathcal{U}\left[\varrho_{0}, \mathbf{m}_{0}, E_{0}\right]
$$

We remark that the value $E\left(T_{-}\right)$in $(\mathbf{A 3})$ and $(\mathbf{A 4})$ can be replaced by

$$
\mathcal{E}=\eta E(T-)+(1-\eta) E(T+),
$$

where $\eta \in[0,1]$ is given, since in this case $E(T+) \leq \mathcal{E} \leq E(T-)$.

\section{Semiflow selection}

The arguments presented below are the same as in [3]. We repeat them here in detail for reader's convenience. We consider the family of functionals

$$
I_{\lambda, F}[\varrho, \mathbf{m}, E]=\int_{0}^{\infty} \mathrm{e}^{-\lambda t} F(\varrho(t), \mathbf{m}(t), E(t)) \mathrm{d} t, \quad \lambda>0,
$$

where $F: X=W^{-\ell, 2}(\Omega) \times W^{-\ell, 2}\left(\Omega ; \mathbb{R}^{N}\right) \times \mathbb{R} \rightarrow \mathbb{R}^{N}$ is a bounded and continuous functional. 
Given $I_{\lambda, F}$ and a set-valued mapping $\mathcal{U}$ we define a selection mapping $I_{\lambda, F} \circ \mathcal{U}$, by

$$
\begin{aligned}
& I_{\lambda, F} \circ \mathcal{U}\left[\varrho_{0}, \mathbf{m}_{0}, E_{0}\right] \\
& \quad=\left\{[\varrho, \mathbf{m}, E] \in \mathcal{U}\left[\varrho_{0}, \mathbf{m}_{0}, E_{0}\right] \mid I_{\lambda, F}[\varrho, \mathbf{m}, E]\right. \\
& \left.\quad \leq I_{\lambda, F}[\tilde{\varrho}, \tilde{\mathbf{m}}, \tilde{E}] \text { for all }[\tilde{\varrho}, \tilde{\mathbf{m}}, \tilde{E}] \in \mathcal{U}\left[\varrho_{0}, \mathbf{m}_{0}, E_{0}\right]\right\}
\end{aligned}
$$

In other words, the selection is choosing minima of the functional $I_{\lambda, F}$. Note that a minimum exists since $I_{\lambda, F}$ is continuous on $Q$ and the set $\mathcal{U}\left[\varrho_{0},(\varrho \mathbf{u})_{0}, E_{0}\right]$ is compact in $Q$. We obtain the following result for the set $I_{\lambda, F} \circ \mathcal{U}$.

Proposition 4.1. Let $\lambda>0$ and $F$ be a bounded continuous functional on $X$. Let

$$
\mathcal{U}:\left[\varrho_{0}, \boldsymbol{m}_{0}, E_{0}\right] \in D \mapsto \mathcal{U}\left[\varrho_{0}, \boldsymbol{m}_{0}, E_{0}\right] \subset 2^{Q}
$$

be a multi-valued mapping having the properties $(\boldsymbol{A 1})-(\mathbf{A 4})$. Then, the map $I_{\lambda, F} \circ \mathcal{U}$ enjoys (A1)-(A4) as well.

Proof. (A1) We proved that $\mathcal{U}\left[\varrho_{0}, \mathbf{m}_{0}, E_{0}\right] \neq \emptyset$ for every $\left[\varrho_{0}, \mathbf{m}_{0}, E_{0}\right] \in D$, and thus $I_{\lambda, F} \circ \mathcal{U}\left[\varrho_{0}, \mathbf{m}_{0}, E_{0}\right] \neq \emptyset$. Since $I_{\lambda, F}: \mathcal{U}\left[\varrho_{0}, \mathbf{m}_{0}, E_{0}\right] \rightarrow \mathbb{R}$ is continuous and since the set of minima of a continuous function is closed (it is the counterpart of a point), we get that $I_{\lambda, F} \circ \mathcal{U}\left[\varrho_{0}, \mathbf{m}_{0}, E_{0}\right] \subseteq \mathcal{U}\left[\varrho_{0}, \mathbf{m}_{0}, E_{0}\right]$ is closed in a compact set and hence compact itself.

(A2) Notice that, since $I_{\lambda, F} \circ \mathcal{U}\left[\varrho_{0}, \mathbf{m}_{0}, E_{0}\right]$ is a compact subset of the separable metric space $Q$ for any $\left[\varrho_{0}, \mathbf{m}_{0}, E_{0}\right] \in D$, the Borel measurability of the multivalued mapping

$$
\left[\varrho_{0}, \mathbf{m}_{0}, E_{0}\right] \in D \mapsto I_{\lambda, F} \circ \mathcal{U}\left[\varrho_{0}, \mathbf{m}_{0}, E_{0}\right] \in \mathcal{K} \subset 2^{Q}
$$

corresponds to measurability with respect to the Hausdorff metric on the space of all compact subsets of Q.

In other words, let $d_{H}$ be the Hausdorff metric on the subspace $\mathcal{K} \subset 2^{Q}$ of all the compact subsets of $Q$ :

$d_{H}\left(K_{1}, K_{2}\right)=\inf _{\varepsilon \geq 0}\left\{K_{1} \subset V_{\varepsilon}\left(K_{2}\right)\right.$ and $\left.K_{2} \subset V_{\varepsilon}\left(K_{1}\right)\right\}$ for all $K_{1}, K_{2} \in \mathcal{K}$,

where $V_{\varepsilon}(A)$ is the $\varepsilon$-neighborhood of the set $A$ in the topology of $Q$; then, it is enough to show that the mapping defined for all $K \in \mathcal{K}$ as

$$
\mathcal{I}_{\lambda, F}[K]=\left\{z \in K \mid I_{\lambda, F}(z) \leq I_{\lambda, F}(\tilde{z}) \text { for all } \tilde{z} \in K\right\}=\left\{z \in K \mid \min _{z \in K} I_{\lambda, F}(z)\right\}
$$

is continuous as a mapping on $\mathcal{K}$ endowed with the Hausdorff metric $d_{H}$. In particular, we want to show that if $K_{n} \stackrel{d_{H}}{\longrightarrow} K$ with $K_{n}, K \in \mathcal{K}$ then $\mathcal{I}_{\lambda, F}\left[K_{n}\right] \stackrel{d_{H}}{\longrightarrow} \mathcal{I}_{\lambda, F}[K]$ for $n \rightarrow \infty$. More precisely, it is enough to show that for every $\varepsilon>0$ there exists $n_{0}=n_{0}(\varepsilon)$ such that

$$
\mathcal{I}_{\lambda, F}\left[K_{n}\right] \subset V_{\varepsilon}\left(\mathcal{I}_{\lambda, F}[K]\right) \text { and } \mathcal{I}_{\lambda, F}[K] \subset V_{\varepsilon}\left(\mathcal{I}_{\lambda, F}\left[K_{n}\right]\right)
$$


for all $n \geq n_{0}$. First of all, notice that by the continuity of $I_{\lambda, F}$ we have

$$
\min _{K_{n}} I_{\lambda, F} \rightarrow \min _{K} I_{\lambda, F} \quad \text { for } n \rightarrow \infty
$$

We start proving the first inclusion of (12). By contradiction, suppose that exists a sequence $\left\{z_{n}\right\}_{n \in \mathbb{N}}$ such that

$$
z_{n} \in K_{n}, \quad I_{\lambda, F}\left(z_{n}\right)=\min _{K_{n}} I_{\lambda, F}, \quad z_{n} \rightarrow z \in K \backslash V_{\varepsilon}\left(\mathcal{I}_{\lambda, F}[K]\right)
$$

in particular, $I_{\lambda, F}(z)>\min _{K} I_{\lambda, F}$. By the continuity of $I_{\lambda, F}$ we have

$$
\min _{K_{n}} I_{\lambda, F}=I_{\lambda, F}\left(z_{n}\right) \rightarrow I_{\lambda, F}(z)>\min _{K} I_{\lambda, F} \quad \text { for } n \rightarrow \infty
$$

but this contradicts (13). Interchanging the roles of $K_{n}$ and $K$ we get the opposite inclusion in (12). We get the claim.

(A3) We want to prove the shift invariance: for every $\left[\varrho_{0}, \mathbf{m}_{0}, E_{0}\right] \in D$ and $[\varrho, \mathbf{m}, E] \in$ $I_{\lambda, F} \circ \mathcal{U}\left[\varrho_{0}, \mathbf{m}_{0}, E_{0}\right]$,

$S_{T} \circ[\varrho, \mathbf{m}, E] \in I_{\lambda, F} \circ \mathcal{U}[\varrho(T), \mathbf{m}(T), E(T-)]$ for any $T>0$.

Let $\left[\varrho^{T}, \mathbf{m}^{T}, E^{T}\right] \in I_{\lambda, F} \circ \mathcal{U}[\varrho(T), \mathbf{m}(T), E(T-)]$; then, since in particular

$$
\begin{aligned}
{[\varrho, \mathbf{m}, E] } & \in \mathcal{U}\left[\varrho_{0}, \mathbf{m}_{0}, E_{0}\right], \\
{\left[\varrho^{T}, \mathbf{m}^{T}, E^{T}\right] } & \in \mathcal{U}[\varrho(T), \mathbf{m}(T), E(T-)],
\end{aligned}
$$

and since $\mathcal{U}$ satisfies property (A4), we get

$$
[\varrho, \mathbf{m}, E] \cup_{T}\left[\varrho^{T}, \mathbf{m}^{T}, E^{T}\right] \in \mathcal{U}\left[\varrho_{0}, \mathbf{m}_{0}, E_{0}\right] .
$$

From the choice of $[\varrho, \mathbf{m}, E]$, which minimize $I_{\lambda, F}$ on $\mathcal{U}\left[\varrho_{0}, \mathbf{m}_{0}, E_{0}\right]$, we obtain

$$
I_{\lambda, F}[\varrho, \mathbf{m}, E] \leq I_{\lambda, F}\left([\varrho, \mathbf{m}, E] \cup_{T}\left[\varrho^{T}, \mathbf{m}^{T}, E^{T}\right]\right)
$$

Hence, using (14) in the fifth line and the definition of $\cup_{T}$ in the sixth line

$$
\begin{aligned}
& I_{\lambda, F}\left(S_{T} \circ[\varrho, \mathbf{m}, E]\right)=\int_{0}^{\infty} \mathrm{e}^{-\lambda t} F\left(S_{T} \circ[\varrho, \mathbf{m}, E](t)\right) \mathrm{d} t \\
& =\int_{0}^{\infty} \mathrm{e}^{-\lambda t} F([\varrho, \mathbf{m}, E](t+T)) \mathrm{d} t \\
& =\mathrm{e}^{\lambda T} \int_{T}^{\infty} \mathrm{e}^{-\lambda s} F([\varrho, \mathbf{m}, E](s)) \mathrm{d} s \\
& =\mathrm{e}^{\lambda T}\left(I_{\lambda, F}[\varrho, \mathbf{m}, E]-\int_{0}^{T} \mathrm{e}^{-\lambda s} F([\varrho, \mathbf{m}, E](s)) \mathrm{d} s\right) \\
& \leq \mathrm{e}^{\lambda T}\left(I_{\lambda, F}\left([\varrho, \mathbf{m}, E] \cup \cup_{T}\left[\varrho^{T}, \mathbf{m}^{T}, E^{T}\right]\right)\right.
\end{aligned}
$$




$$
\begin{aligned}
& \left.-\int_{0}^{T} \mathrm{e}^{-\lambda s} F([\varrho, \mathbf{m}, E](s)) \mathrm{d} s\right) \\
= & \mathrm{e}^{\lambda T} \int_{T}^{\infty} \mathrm{e}^{-\lambda s} F\left(\left[\varrho^{T}, \mathbf{m}^{T}, E^{T}\right](s-T)\right) \mathrm{d} s \\
= & \mathrm{e}^{\lambda T} \int_{0}^{\infty} \mathrm{e}^{-\lambda(t+T)} F\left(\left[\varrho^{T}, \mathbf{m}^{T}, E^{T}\right](t)\right) \mathrm{d} t \\
= & I_{\lambda, F}\left[\varrho^{T}, \mathbf{m}^{T}, E^{T}\right] .
\end{aligned}
$$

This implies that $S_{T} \circ[\varrho, \mathbf{m}, E]$ minimizes $I_{\lambda, F}$ and consequently belongs to $I_{\lambda, F} \circ \mathcal{U}[\varrho(T), \mathbf{m}(T), E(T-)]$ for any $T>0$.

(A4) We want to prove the continuation: if $T>0$ and $\left[\varrho^{1}, \mathbf{m}^{1}, E^{1}\right] \in I_{\lambda, F} \circ$ $\mathcal{U}\left[\varrho_{0}, \mathbf{m}_{0}, E_{0}\right],\left[\varrho^{2}, \mathbf{m}^{2}, E^{2}\right] \in I_{\lambda, F} \circ \mathcal{U}\left[\varrho^{1}(T), \mathbf{m}^{1}(T), E^{1}(T-)\right]$, then

$$
\left[\varrho^{1}, \mathbf{m}^{1}, E^{1}\right] \cup_{T}\left[\varrho^{2}, \mathbf{m}^{2}, E^{2}\right] \in I_{\lambda, F} \circ \mathcal{U}\left[\varrho_{0}, \mathbf{m}_{0}, E_{0}\right] .
$$

Using the shift invariance for $\mathcal{U}$, we obtain

$$
S_{T} \circ\left[\varrho^{1}, \mathbf{m}^{1}, E^{1}\right] \in \mathcal{U}\left[\varrho^{1}(T), \mathbf{m}^{1}(T), E^{1}(T-)\right] ;
$$

since $\left[\varrho^{2}, \mathbf{m}^{2}, E^{2}\right]$ is a minimum of $I_{\lambda, F}$ on $\mathcal{U}\left[\varrho^{1}(T), \mathbf{m}^{1}(T), E^{1}(T-)\right]$, we get

$$
I_{\lambda, F}\left[\varrho^{2}, \mathbf{m}^{2}, E^{2}\right] \leq I_{\lambda, F}\left(S_{T} \circ\left[\varrho^{1}, \mathbf{m}^{1}, E^{1}\right]\right) .
$$

Hence, using (15) in the fourth line

$$
\begin{aligned}
I_{\lambda, F} & \left(\left[\varrho^{1}, \mathbf{m}^{1}, E^{1}\right] \cup_{T}\left[\varrho^{2}, \mathbf{m}^{2}, E^{2}\right]\right) \\
& =\int_{0}^{T} \mathrm{e}^{-\lambda t} F\left(\left[\varrho^{1}, \mathbf{m}^{1}, E^{1}\right](t)\right) \mathrm{d} t+\int_{T}^{\infty} \mathrm{e}^{-\lambda t} F\left(\left[\varrho^{2}, \mathbf{m}^{2}, E^{2}\right](t-T)\right) \mathrm{d} t \\
& =\int_{0}^{T} \mathrm{e}^{-\lambda t} F\left(\left[\varrho^{1}, \mathbf{m}^{1}, E^{1}\right](t)\right) \mathrm{d} t+\mathrm{e}^{-\lambda T} \int_{0}^{\infty} \mathrm{e}^{-\lambda s} F\left(\left[\varrho^{2}, \mathbf{m}^{2}, E^{2}\right](s)\right) \mathrm{d} s \\
& =\int_{0}^{T} \mathrm{e}^{-\lambda t} F\left(\left[\varrho^{1}, \mathbf{m}^{1}, E^{1}\right](t)\right) \mathrm{d} t+\mathrm{e}^{-\lambda T} I_{\lambda, F}\left[\varrho^{2}, \mathbf{m}^{2}, E^{2}\right] \\
& \leq \int_{0}^{T} \mathrm{e}^{-\lambda t} F\left(\left[\varrho^{1}, \mathbf{m}^{1}, E^{1}\right](t)\right) \mathrm{d} t+\mathrm{e}^{-\lambda T} I_{\lambda, F}\left(S_{T} \circ\left[\varrho^{1}, \mathbf{m}^{1}, E^{1}\right]\right) \\
& =\int_{0}^{T} \mathrm{e}^{-\lambda t} F\left(\left[\varrho^{1}, \mathbf{m}^{1}, E^{1}\right](t)\right) \mathrm{d} t+\mathrm{e}^{-\lambda T} \int_{0}^{\infty} \mathrm{e}^{-\lambda s} F\left(\left[\varrho^{1}, \mathbf{m}^{1}, E^{1}\right](s+T)\right) \mathrm{d} s \\
& =\int_{0}^{T} \mathrm{e}^{-\lambda t} F\left(\left[\varrho^{1}, \mathbf{m}^{1}, E^{1}\right](t)\right) \mathrm{d} t+\int_{T}^{\infty} \mathrm{e}^{-\lambda t} F\left(\left[\varrho^{1}, \mathbf{m}^{1}, E^{1}\right](t)\right) \mathrm{d} t \\
& =I_{\lambda, F}\left[\varrho^{1}, \mathbf{m}^{1}, E^{1}\right] .
\end{aligned}
$$

Using the continuation property for $\mathcal{U}$, we have that

$$
\left[\varrho^{1}, \mathbf{m}^{1}, E^{1}\right] \cup_{T}\left[\varrho^{2}, \mathbf{m}^{2}, E^{2}\right] \in \mathcal{U}\left[\varrho_{0}, \mathbf{m}_{0}, E_{0}\right],
$$

and since $\left[\varrho^{1}, \mathbf{m}^{1}, E^{1}\right]$ is a minimum of $I_{\lambda, F}$, we must have

$$
I_{\lambda, F}\left(\left[\varrho^{1}, \mathbf{m}^{1}, E^{1}\right] \cup_{T}\left[\varrho^{2}, \mathbf{m}^{2}, E^{2}\right]\right)=I_{\lambda, F}\left[\varrho^{1}, \mathbf{m}^{1}, E^{1}\right] ;
$$


thus $\left[\varrho^{1}, \mathbf{m}^{1}, E^{1}\right] \cup_{T}\left[\varrho^{2}, \mathbf{m}^{2}, E^{2}\right] \in I_{\lambda, F} \circ \mathcal{U}\left[\varrho_{0}, \mathbf{m}_{0}, E_{0}\right]$.

\subsection{Selection sequence}

First of all, we will select only those solutions that are admissible, meaning minimal with respect to the relation $\prec$ introduced in Definition 1.5. To this end we consider the functional $I_{1, \beta}$ with

$\beta(\varrho, \mathbf{m}, E)=\beta(E), \quad \beta: \mathbb{R} \rightarrow \mathbb{R}$ smooth, bounded, and strictly increasing.

Lemma 4.2. Suppose that $[\varrho, \boldsymbol{m}, E] \in \mathcal{U}\left[\varrho_{0}, \boldsymbol{m}_{0}, E_{0}\right]$ satisfies

$$
\int_{0}^{\infty} \mathrm{e}^{-t} \beta(E(t)) \mathrm{d} t \leq \int_{0}^{\infty} \mathrm{e}^{-t} \beta(\tilde{E}(t)) \mathrm{d} t
$$

for any $[\tilde{\varrho}, \tilde{\boldsymbol{m}}, \tilde{E}] \in \mathcal{U}\left[\varrho_{0}, \boldsymbol{m}_{0}, E_{0}\right]$. Then, $[\varrho, \boldsymbol{m}, E]$ is $\prec$ minimal, meaning, admissible.

Proof. We proceed by contradiction. Let $[\tilde{\varrho}, \tilde{\mathbf{m}}, \tilde{E}] \in \mathcal{U}\left[\varrho_{0}, \mathbf{m}_{0}, E_{0}\right]$ be such that $[\tilde{\varrho}, \tilde{\mathbf{m}}, \tilde{E}] \prec[\varrho, \mathbf{m}, E]$, that is, $\tilde{E} \leq E$ in $[0, \infty)$. Then, since $\beta$ is strictly increasing, $\beta(\tilde{E}(t)) \leq \beta(E(t))$ for every $t \in[0, \infty)$, which implies that $\mathrm{e}^{-t}[\beta(E(t))-$ $\beta(\tilde{E}(t))] \geq 0$. Using the monotonicity of the integral, we obtain

$$
\int_{0}^{\infty} \mathrm{e}^{-t}[\beta(E(t))-\beta(\tilde{E}(t))] \mathrm{d} t \geq 0 ;
$$

on the other side, condition (16) tells us that

$$
\int_{0}^{\infty} \mathrm{e}^{-t}[\beta(E(t))-\beta(\tilde{E}(t))] \mathrm{d} t \leq 0 .
$$

The only possibility is to have the equality in both the integral relations above and thus $\beta(E(t))=\beta(\tilde{E}(t))$ for a.e. $t \in(0, \infty)$; since $\beta$ is strictly increasing, this implies $E=\tilde{E}$ a.e. in $(0, \infty)$.

We will need this topological result, which is a variation of the Cantor's intersection theorem.

Theorem 4.3. Let $S$ be a Hausdorff space. A decreasing nested sequence of non-empty compact subsets of $S$ is non-empty. In other words, supposing $\left\{C_{k}\right\}_{k \in \mathbb{N}}$ is a sequence of non-empty compact subsets of $S$ satisfying

$$
C_{0} \supseteq C_{1} \supseteq C_{2} \supseteq \cdots \supseteq C_{k} \supseteq \cdots
$$

it follows that

$$
\bigcap_{k \in \mathbb{N}} C_{k} \neq \emptyset
$$


Proof. By contradiction, assume $\bigcap_{k \in \mathbb{N}} C_{k}=\emptyset$. For each $n$, let $U_{n}=C_{0} \backslash C_{n}$; since

$$
\bigcup_{n \in \mathbb{N}} U_{n}=\bigcup_{n \in \mathbb{N}}\left(C_{0} \backslash C_{n}\right)=C_{0} \backslash\left(\bigcap_{n \in \mathbb{N}} U_{n}\right)
$$

and $\bigcap_{n \in \mathbb{N}} C_{n}=\emptyset$, we obtain $\bigcup_{n \in \mathbb{N}} U_{n}=C_{0}$. Since $C_{0} \subset S$ is compact and $\left\{U_{n}\right\}_{n \in \mathbb{N}}$ is an open cover (on $C_{0}$ ) of $C_{0}$, we can extract a finite cover $\left\{U_{n_{1}}, \ldots, U_{n_{m}}\right\}$. Let $U_{k}$ be the largest set of this cover ( $C_{k}$ the correspondent smallest set), which exists by the ordering hypothesis on the collection $\left\{C_{n}\right\}_{n \in \mathbb{N}}$. Then,

$$
C_{0} \subset \bigcup_{j=1}^{m} U_{n_{j}}=\bigcup_{j=1}^{m}\left(C_{0} \backslash C_{n_{j}}\right)=C_{0} \backslash \bigcap_{j=1}^{m} C_{n_{j}}=C_{0} \backslash C_{k}=U_{k} .
$$

Then, $C_{k}=C_{0} \backslash U_{k}=\emptyset$, a contradiction since every set of the sequence $\left\{C_{n}\right\}_{n \in \mathbb{N}}$ is non-empty by hypothesis.

We are now ready to prove Theorem 2.2.

Proof of Theorem 2.2. Selecting $I_{1, \beta} \circ \mathcal{U}$ from $\mathcal{U}$ we know from Lemma 4.2 that the new selection contains only admissible solutions for any $\left[\varrho_{0}, \mathbf{m}_{0}, E_{0}\right] \in D$.

Next, we choose a countable basis $\left\{\mathbf{e}_{n}\right\}_{n \in \mathbb{N}}$ in $L^{2}\left(\Omega ; \mathbb{R}^{N}\right)$, and a countable set $\left\{\lambda_{k}\right\}_{k \in \mathbb{N}}$ which is dense in $(0, \infty)$. We consider a countable family of functionals,

$$
\begin{aligned}
& I_{k, 0}[\varrho, \mathbf{m}, E]=\int_{0}^{\infty} \mathrm{e}^{-\lambda_{k} t} \beta(E(t)) \mathrm{d} t, \\
& I_{k, n}[\varrho, \mathbf{m}, E]=\int_{0}^{\infty} \mathrm{e}^{-\lambda_{k} t} \beta\left(\int_{\Omega} \mathbf{m}(t, \cdot) \cdot \mathbf{e}_{n} \mathrm{~d} x\right) \mathrm{d} t ;
\end{aligned}
$$

the functionals are well defined since $\mathbf{m}(t, \cdot) \in W^{-\ell, 2}\left(\Omega ; \mathbb{R}^{N}\right)$ for all $t$. Let $\{(k(j), n(j))\}_{j=1}^{\infty}$ be an enumeration of all the involved combinations of indices, that is, an enumeration of the countable set

$$
(\mathbb{N} \times\{0\}) \cup(\mathbb{N} \times \mathbb{N}) .
$$

We define

$$
\mathcal{U}^{j}=I_{k(j), n(j)} \circ \cdots \circ I_{k(1), n(1)} \circ I_{1, \beta} \circ \mathcal{U}, \quad j=1,2, \ldots,
$$

and

$$
\mathcal{U}^{\infty}=\bigcap_{j=1}^{\infty} \mathcal{U}^{j}
$$

The set-valued mapping

$$
\left[\varrho_{0}, \mathbf{m}_{0}, E_{0}\right] \in D \mapsto \mathcal{U}^{\infty}\left[\varrho_{0}, \mathbf{m}_{0}, E_{0}\right]
$$

enjoys the properties (A1)-(A4). Indeed: 
(A1) first, notice that for every fixed initial data $\left[\varrho_{0}, \mathbf{m}_{0}, E_{0}\right] \in D$ the sets $\mathcal{U}^{j}\left[\varrho_{0}, \mathbf{m}_{0}\right.$, $\left.E_{0}\right]$ are nested:

$I_{1, \beta} \circ \mathcal{U}\left[\varrho_{0}, \mathbf{m}_{0}, E_{0}\right] \supseteq \mathcal{U}^{1}\left[\varrho_{0}, \mathbf{m}_{0}, E_{0}\right] \supseteq \ldots \supseteq \mathcal{U}^{j}\left[\varrho_{0}, \mathbf{m}_{0}, E_{0}\right] \supseteq \ldots$

By Proposition 4.1 we can deduce that $I_{1, \beta} \circ \mathcal{U}\left[\varrho_{0}, \mathbf{m}_{0}, E_{0}\right]$ is compact, and iterating this procedure we obtain that all $\mathcal{U}^{j}\left[\varrho_{0}, \mathbf{m}_{0}, E_{0}\right]$ are compact. Since $Q$ is a Hausdorff space, every compact set is also closed and a countable intersection of closed set is closed. Since $\mathcal{U}^{\infty}\left[\varrho_{0}, \mathbf{m}_{0}, E_{0}\right] \subseteq I_{1, \beta} \circ \mathcal{U}\left[\varrho_{0}, \mathbf{m}_{0}, E_{0}\right]$, which is compact, we obtain that $\mathcal{U}^{\infty}\left[\varrho_{0}, \mathbf{m}_{0}, E_{0}\right]$ is compact. By Proposition 4.1 we can also deduce that every $\mathcal{U}^{j}\left[\varrho_{0}, \mathbf{m}_{0}, E_{0}\right]$ is non-empty; applying Theorem 4.3 we then get that $\mathcal{U}^{\infty}\left[\varrho_{0}, \mathbf{m}_{0}, E_{0}\right] \neq \emptyset$;

(A2) as it is an intersection set-valued map obtained from measurable set-valued maps, it is also measurable;

(A3) to prove the shift property, let $\left[\varrho_{0}, \mathbf{m}_{0}, E_{0}\right] \in D$ and $[\varrho, \mathbf{m}, E] \in \mathcal{U}^{\infty}\left[\varrho_{0}, \mathbf{m}_{0}, E_{0}\right]$; then, in particular $[\varrho, \mathbf{m}, E] \in \mathcal{U}^{j}\left[\varrho_{0}, \mathbf{m}_{0}, E_{0}\right]$ for every $j$. By Proposition 4.1, we can deduce that $I_{1, \beta} \circ \mathcal{U}$ satisfies the shift invariance property, and iterating this procedure we obtain that this holds for every $\mathcal{U}^{j}$. This implies

$S_{T} \circ[\varrho, \mathbf{m}, E] \in \mathcal{U}^{j}[\varrho(T), \mathbf{m}(T), E(T-)]$, for all $j$ and all $T>0$.

Thus,

$$
S_{T} \circ[\varrho, \mathbf{m}, E] \in \mathcal{U}^{\infty}[\varrho(T), \mathbf{m}(T), E(T-)], \text { for all } T>0
$$

(A4) to prove the continuation property, let $T>0,\left[\varrho^{1}, \mathbf{m}^{1}, E^{1}\right] \in \mathcal{U}^{\infty}\left[\varrho_{0}, \mathbf{m}_{0}, E_{0}\right]$ and $\left[\varrho^{2}, \mathbf{m}^{2}, E^{2}\right] \in \mathcal{U}^{\infty}\left[\varrho^{1}(T), \mathbf{m}^{1}(T), E^{1}(T-)\right]$; then, in particular we have $\left[\varrho^{1}, \mathbf{m}^{1}, E^{1}\right] \in \mathcal{U}^{j}\left[\varrho_{0}, \mathbf{m}_{0}, E_{0}\right]$ and $\left[\varrho^{2}, \mathbf{m}^{2}, E^{2}\right] \in \mathcal{U}^{j}\left[\varrho^{1}(T), \mathbf{m}^{1}(T), E^{1}(T-)\right]$ for every $j$. By Proposition 4.1, we can deduce that $I_{1, \beta} \circ \mathcal{U}$ satisfies the continuation property, and iterating this procedure we obtain that this holds for every $\mathcal{U}^{j}$. This implies

$\left[\varrho^{1}, \mathbf{m}^{1}, E^{1}\right] \cup_{T}\left[\varrho^{2}, \mathbf{m}^{2}, E^{2}\right] \in \mathcal{U}^{j}\left[\varrho_{0}, \mathbf{m}_{0}, E_{0}\right]$ for all $j$ and all $T>0$.

Thus,

$$
\left[\varrho^{1}, \mathbf{m}^{1}, E^{1}\right] \cup_{T}\left[\varrho^{2}, \mathbf{m}^{2}, E^{2}\right] \in \mathcal{U}^{\infty}\left[\varrho_{0}, \mathbf{m}_{0}, E_{0}\right] \text { for all } T>0 .
$$

We claim that for every $\left[\varrho_{0}, \mathbf{m}_{0}, E_{0}\right] \in D$ the set $\mathcal{U}^{\infty}$ is a singleton, meaning

$$
\mathcal{U}^{\infty}\left[\varrho_{0}, \mathbf{m}_{0}, E_{0}\right]=U\left\{\varrho_{0}, \mathbf{m}_{0}, E_{0}\right\} \in Q .
$$

To verify this, we observe that

$$
I_{k(j), n(j)}\left[\varrho^{1}, \mathbf{m}^{1}, E^{1}\right]=I_{k(j), n(j)}\left[\varrho^{2}, \mathbf{m}^{2}, E^{2}\right]
$$


for any $\left[\varrho^{1}, \mathbf{m}^{1}, E^{1}\right],\left[\varrho^{2}, \mathbf{m}^{2}, E^{2}\right] \in \mathcal{U}^{\infty}\left[\varrho_{0}, \mathbf{m}_{0}, E_{0}\right]$ for all $j=1,2, \ldots$; from the choice of $\{k(j), n(j)\}_{j \in \mathbb{N}}$, we can see the integrals $I_{k(j), n(j)}$ as Laplace transforms

$$
F\left(\lambda_{k}\right)=\int_{0}^{\infty} \mathrm{e}^{-\lambda_{k} t} f(t) \mathrm{d} t
$$

of the functions

$$
f \in\left\{\beta(E), \beta\left(\int_{\Omega} \mathbf{m} \cdot \mathbf{e}_{n} \mathrm{~d} x\right)\right\} .
$$

We can apply Lerch's theorem: if a function $F$ has the inverse Laplace transform $f$, then $f$ is uniquely determined (considering functions which differ from each other only on a point set having Lebesgue measure zero as the same). Then, we get that

$$
\begin{aligned}
\beta\left(E^{1}(t)\right) & =\beta\left(E^{2}(t)\right), \\
\beta\left(\int_{\Omega} \mathbf{m}^{1}(t, \cdot) \cdot \mathbf{e}_{n} \mathrm{~d} x\right) & =\beta\left(\int_{\Omega} \mathbf{m}^{2}(t, \cdot) \cdot \mathbf{e}_{n} \mathrm{~d} x\right),
\end{aligned}
$$

for all $n \in \mathbb{N}$ and for a.e. $t \in(0, \infty)$. As $\beta$ is strictly increasing, we must in particular have

$$
E^{1}(t)=E^{2}(t), \quad\left\langle\mathbf{m}^{1}(t, \cdot) ; \mathbf{e}_{n}\right\rangle_{L^{2}\left(\Omega ; \mathbb{R}^{N}\right)}=\left\langle\mathbf{m}^{2}(t, \cdot) ; \mathbf{e}_{n}\right\rangle_{L^{2}\left(\Omega ; \mathbb{R}^{N}\right)},
$$

for all $n \in \mathbb{N}$ and for a.e. $t \in(0, \infty)$. Since $\left\{\mathbf{e}_{n}\right\}_{n \in \mathbb{N}}$ form a basis in $L^{2}\left(\Omega ; \mathbb{R}^{N}\right)$, we conclude

$$
\mathbf{m}^{1}=\mathbf{m}^{2} \text {, and } E^{1}=E^{2} \text { a.e. on }(0, \infty)
$$

From the continuity equation (1) and from the fact that $\varrho^{1}(0, \cdot)=\varrho^{2}(0, \cdot)$ it is easy to see that

$$
\varrho^{1}=\varrho^{2} \text { a.e. }(0, \infty)
$$

It remains to prove that $U$ is a semiflow selection: measurability follows from (A2), while the semigroup property follows from (A3): for $t_{1}, t_{2} \geq 0$ it holds

$U\left\{\varrho_{0}, \mathbf{m}_{0}, E_{0}\right\}\left(t_{1}+t_{2}\right)=S_{t_{1}} \circ U\left\{\varrho_{0}, \mathbf{m}_{0}, E_{0}\right\}\left(t_{2}\right)=U\left\{\varrho\left(t_{1}\right), \mathbf{m}\left(t_{1}\right), E\left(t_{1}-\right)\right\}\left(t_{2}\right)$.

This completes the proof.

Remark 4.4. In comparison with [3], it is worth noticing that the family of functionals defined in (17) do not depend on the density $\varrho$ : they are not necessary since, once the uniqueness of the momenta is achieved, the one of the densities easily follows from the continuity equation (1). 


\section{Restriction to semigroup acting only on the initial data}

As a matter of fact, the semiflow selection $U=U\left\{\varrho_{0}, \mathbf{m}_{0}, E_{0}\right\}$ is determined in terms of the three state variables: the density $\varrho_{0}$, the momentum $\mathbf{m}_{0}$, and the energy $E_{0}$. Introduction of the energy might be superfluous; indeed, as pointed out in (10)

$$
E(\tau)=\int_{\Omega}\left[\frac{1}{2} \frac{|\mathbf{m}|^{2}}{\varrho}+P(\varrho)\right](\tau, \cdot) \mathrm{d} x \text { for a.e. } \tau \geq 0 .
$$

The point is that the equality holds with the exception of a zero measure set of times. More specifically, the energy $E(\tau)$ is a non-increasing function with well-defined right and left limits $E(\tau \pm)$, while

$$
\int_{\Omega}\left[\frac{1}{2} \frac{|\mathbf{m}|^{2}}{\varrho}+P(\varrho)\right](\tau, \cdot) \mathrm{d} x
$$

is defined at any $\tau$ in terms of weakly continuous functions $t \mapsto \varrho(t, \cdot), t \mapsto \mathbf{m}(t, \cdot)$. Due to the convexity of the superposition

$$
[\varrho, \mathbf{m}] \mapsto \frac{1}{2} \frac{|\mathbf{m}|^{2}}{\varrho}+P(\varrho)
$$

the function

$$
\tau \mapsto \int_{\Omega}\left[\frac{1}{2} \frac{|\mathbf{m}|^{2}}{\varrho}+P(\varrho)\right](\tau, \cdot) \mathrm{d} x
$$

is lower semi-continuous in $\tau$. In particular,

$$
\int_{\Omega}\left[\frac{1}{2} \frac{|\mathbf{m}|^{2}}{\varrho}+P(\varrho)\right](\tau, \cdot) \mathrm{d} x \leq E(\tau \pm) \text { for any } \tau,
$$

where equality holds with the exception of a set of times of measure zero.

We may introduce a new selection defined only in terms of the initial data $\varrho_{0}, \mathbf{m}_{0}$; however, the price to pay is that the semigroup property will hold almost everywhere in time. More specifically, we can state this final result.

Theorem 5.1. Let $U=U\left\{\varrho_{0}, \boldsymbol{m}_{0}, E_{0}\right\}$ be the semiflow selection associated with the Navier-Stokes system in the sense of Definition 2.1. Consider the set of initial data

$$
\widetilde{D}=\left\{\left[\varrho_{0}, \boldsymbol{m}_{0}\right]:\left[\varrho_{0}, \boldsymbol{m}_{0}, \int_{\Omega}\left(\frac{1}{2} \frac{\left|\boldsymbol{m}_{0}\right|^{2}}{\varrho_{0}}+P\left(\varrho_{0}\right)\right) \mathrm{d} x\right] \in D\right\} .
$$

Defining $V: \widetilde{D} \rightarrow Q$ such that

$$
V\left\{\varrho_{0}, \boldsymbol{m}_{0}\right\}(t)=U\left\{\varrho_{0}, \boldsymbol{m}_{0}, \int_{\Omega}\left[\frac{1}{2} \frac{\left|\boldsymbol{m}_{0}\right|^{2}}{\varrho_{0}}+P\left(\varrho_{0}\right)\right] \mathrm{d} x\right\}(t)
$$


for all $t \in(0, \infty)$, then $V$ will satisfy the semigroup property only almost everywhere; more precisely, calling $\mathcal{T} \subset(0, \infty)$ the set of times defined as

$$
\mathcal{T}=\left\{\tau \in(0, \infty): E(\tau)=\int_{\Omega}\left[\frac{1}{2} \frac{|\boldsymbol{m}|^{2}}{\varrho}+P(\varrho)\right](\tau, \cdot) \mathrm{d} x\right\},
$$

then $\mathcal{T}$ is a set of full measure and

$$
V\left\{\varrho_{0}, \boldsymbol{m}_{0}\right\}\left(t_{1}+t_{2}\right)=V\left\{V\left\{\varrho_{0}, \boldsymbol{m}_{0}\right\}\left(t_{1}\right)\right\}\left(t_{2}\right)
$$

holds for all $t_{1}, t_{2} \in \mathcal{T}$.

\section{Acknowledgements}

Open Access funding provided by Projekt DEAL. This work was supported by the Einstein Foundation, Berlin. The author wishes to thank Prof. Eduard Feireisl for the helpful advice and discussions.

Open Access. This article is licensed under a Creative Commons Attribution 4.0 International License, which permits use, sharing, adaptation, distribution and reproduction in any medium or format, as long as you give appropriate credit to the original author(s) and the source, provide a link to the Creative Commons licence, and indicate if changes were made. The images or other third party material in this article are included in the article's Creative Commons licence, unless indicated otherwise in a credit line to the material. If material is not included in the article's Creative Commons licence and your intended use is not permitted by statutory regulation or exceeds the permitted use, you will need to obtain permission directly from the copyright holder. To view a copy of this licence, visit http://creativecommons.org/licenses/ by $/ 4.0 /$.

Publisher's Note Springer Nature remains neutral with regard to jurisdictional claims in published maps and institutional affiliations.

\section{REFERENCES}

[1] D. Breit, E. Feireisl and M. Hofmanová, Dissipative Solutions and Semiflow Selection for the Complete Euler System, Communications in Mathematical Physics; 2020

[2] D. Breit, E. Feireisl and M. Hofmanová, Markov selection for the stochastic compressible NavierStokes system, arXiv:1809.07265; 2018

[3] D. Breit, E. Feireisl and M. Hofmanová, Solution semiflow to the isentropic Euler system, Archive for Rational Mechanics and Analysis; 2019

[4] J. E. Cardona and L. Kapitanski, Semiflow selection and Markov selection theorems, arXiv: $1707.04778 ; 2017$

[5] N. Chaudhuri, On weak-strong uniqueness for compressible Navier-Stokes system with general pressure laws, Nonlinear Analysis: Real World Applications; 2019

[6] E. Feireis1, Dynamics of Viscous Compressible Fluids, Oxford University Press, Oxford; 2003

[7] F. Flandoli and M. Romito, Markov selections for the 3D stochastics Navier-Stokes equations, Probab. Theory Related Fields, 140(3-4): 407-458; 2008

[8] A. V. Kazhikhov, Correctness "in the large" of mixed boundary value problems for a model system of equations of a viscous gas, Dinamika Splošn. Sredy, (Vyp. 21 Tecenie Zidkost. so Svobod. Granicami), 188:18-47; 1975.

[9] N. V. Krylov, The selection of a Markov process from a Markov system of processes, and the construction of quasidiffusion processes, Izv. Akad. Nauk SSSR Ser. Mat., 37: 691-708; 1973 
[10] P.-L. Lions, Mathematical Topics in Fluid Mechanics, Volume 2: Compressible Models, Oxford Science Publications, Oxford; 1998

\author{
Danica Basarić \\ Institute für Mathematik \\ Technische Universität Berlin \\ Straße des 17. Juni 136 \\ 10623 Berlin \\ Germany \\ E-mail:basaric@math.tu-berlin.de
}

